\title{
Editorial \\ Special issue: Collisions in collisionless plasmas
}

\author{
F. Valentini $\uparrow$ \\ Dipartimento di Fisica, Universitá della Calabria, 87036 Rende (CS), Italy
}

(Received 19 May 2014)

The special issue 'Collisions in collisionless plasmas' is intended to provide a collection of relevant contributions from different scientific fields in plasma physics, including experiments, theory, and numerical modeling, focused on the role of collisions on the dynamical evolution of weakly collisional plasma systems.

The idea of a special issue in the Journal of Plasma Physics completely dedicated to the topic of collisions in plasmas came out from many interesting scientific discussions, occurred in several workshops and conferences in 2013, on turbulent dissipation and heating processes, in systems usually considered collision-free in good approximation.

The evident oxymoron in the title of this special issue gives a clear idea of the scope and the motivations of this scientific effort. Within the framework of the kinetic plasma theory, one often restricts attention to the class of the so-called collisionless plasmas. In most cases, this assumption of weak collisionality is satisfied in good approximation. On the other hand, often the existence of a collisionless limit is erroneously confused with the negligibility of collisional effects, and, forgetting about the physical ranges in which this approximation can be considered valid, particle collisions in most cases just fall by the wayside. Nevertheless, when speaking about plasma dissipation and heating processes, one just cannot fail to take into account collisional effects. In fact, on the basis of the H-theorem, the only way to increase entropy in a plasma, and therefore produce heating in general thermodynamic sense, is via collisions. For instance, in analogy with a fluid with small viscosity, where short scales will form in order for the system to access dissipation and get rid of energy that might be injected into it at large scales, similarly for collisionless plasmas, waveparticle interactions (phase mixing processes, nonlinear couplings, etc.) will produce small-scale structures in velocity space, in such a way collisions can become important and thermalize free energy.

Moreover, many plasma systems, commonly viewed as collisionless, often exhibit remarkably good correlations between various measured quantities and collisional age. As an example, one can think about the solar wind: In the interplanetary medium, in fact, the particle mean free path is typically estimated to be of the order of $1 \mathrm{AU}$. This rough estimation is obtained when assuming that the particle velocity distributions are close to thermodynamic equilibrium. Nevertheless, in such a weak collisional medium, resonant wave-particle interactions can significantly shape the particle distribution functions, creating sharp gradients and local deformations in velocity space, and producing in principle significant departures from Maxwellians.

If one thinks about the general form of the Landau collisional integral, these departures from Maxwellians, and the consequent generation of sharp gradients in

$\dagger$ Email address for correspondence: francesco.valentini@fis.unical.it 
velocity space, should enhance locally the plasma collisionality, and are expected to play a determinant role in the process of plasma thermalization and heating.

The description of realistic plasmas based on the complete Landau model is a big challenge from both analytical and numerical point of view because of the nonlinear nature of the Landau operator and of its multi-dimensionality. A remarkable attempt to attack the full Landau problem has been made by Pareschi et al. (2000), who proposed a numerical algorithm for the Landau collisional integral based on the use of Fast Fourier Transform (FFT) routines. Moreover, the Takizuka-Abe Monte Carlo collisional model, able to reproduce the full Landau integral, is usually employed in particle-in-cell simulations (Takizuka and Abe 1977; Daughton et al. 2009).

A different approach to the problem of plasma collisions consists to mimic the full Landau operator through simplified differential operators of the Fokker-Planck type, such as, for example, the nonlinear Dougherty (1964) operator. The Dougherty operator satisfies the $\mathrm{H}$-theorem and has been derived by requiring the conservation of mass, energy, and momentum, and that a generic Maxwellian is a unique equilibrium solution. At this point, many open questions need answer: What are the minimal ingredients for an adequate collision operator? What are the requirements that a simplified collisional operator must satisfy to model a real plasma? The answer to these questions might be different for a weak collisional regime from that for a strong collisional one. In order to provide correct answers to these questions, much work remains yet to be done.

This special issue has been thought to give a valuable snapshot on the problem of plasma collisions in different scientific areas of plasma physics, encompassing basic plasma physics and its myriad of applications: space and astrophysical plasmas, plasma technology, laboratory plasmas, etc.

I gratefully thank the new editorial board of the Journal of Plasma Physics, and, in particular, Prof. A. Schekochihin, for valuable help and support in the development of this scientific project.

\section{REFERENCES}

Daughton, W., Roytershteyn, V., Albright, B. J., Karimabadi, H., Yin, L. and Bowers, K. J. 2009 Influence of Coulomb collisions on the structure of reconnection layers. Phys. Plasmas 16, 072117.

Dougherty, J. P. 1964 Model Fokker-Planck equation for a plasma and its solution. Phys. Fluids 7, 1788.

Pareschi, L., Russo L. G. and Toscani, G. 2000 Fast spectral methods for the Fokker-Planck-Landau collision operator. J. Comput. Phys. 165, 216-236.

Takizuka, T. and Abe, H. 1977 A binary collision model for plasma simulation with a particle code. J. Comput. Phys. 25, 205-219. 